\title{
A CYCLE IS THE FUNDAMENTAL CLASS OF AN EULER SPACE
}

\author{
R. BENEDETTI AND M. DEDÓ
}

\begin{abstract}
We prove that every cycle in a closed P.L. manifold $M$ can be regarded as the fundamental class of an Euler subpolyhedron of $M$.
\end{abstract}

Let $V$ be a compact real analytic manifold without boundary. It is a long-standing problem to see which $\left(\mathbf{Z}_{2^{-}}\right)$homology classes of $V$ can be represented as the fundamental class of an analytic subset of $V$ (and, in fact, it is conjectured that this is true for any homology class). The analogous problem arises with real algebraic manifolds, although in this case the general statement is false (even if $V$ is connected; see, for instance, [BT]).

D. Sullivan (in [S]) observed that every real analytic set can be regarded as an Euler space (see definition below); it is then natural to ask, first of all, if it is true that every homology class of a closed P.L. manifold $M$ can be represented as the fundamental class of an Euler subpolyhedron of $M$.

In this note we prove that this in fact happens: actually, we give a construction to add lower-dimensional simplexes to a cycle in $M$ until we get an Euler space (in $M$ ).

The techniques used are entirely elementary and involve merely P.L. transversality (as stated for example in [RS]) and combinatorial results on Euler spaces (see [A]).

We shall work in the P.L. category. For notations and definitions we refer to [RS]. All cycles and manifolds are intended unoriented and compact.

By an $n$-cycle $P$ we mean a polyhedron $P=|K|$ such that

(1) $n=\max \{\operatorname{dim} A$, for $A$ a simplex of $K\}$,

(2) each $(n-1)$-simplex of $K$ is the face of an even number of simplexes of $K$.

By an $n$-cycle $P$ with boundary $\partial P$ we mean a pair of polyhedra $(P, \partial P)=|K, \partial K|$ such that (1) $n=\max \{\operatorname{dim} A$, for $A$ a simplex of $K\},(2) \partial P$ is an $(n-1)$-cycle, (3) each $(n-1)$-simplex of $K \backslash \partial K$ is the face of an even number of $n$-simplexes of $K$, (4) each $(n-1)$-simplex of $\partial K$ is the face of an odd number of $n$-simplexes of $K$. A cycle (with boundary) in $M$ is a subpolyhedron of $M$ which is a cycle (with boundary).

A closed (P.L.) manifold is a compact (P.L.) manifold without boundary.

An Euler space is a polyhedron $P$ such that, for each $x \in P, \chi(\operatorname{lk}(x, P)) \equiv 0$ $(\bmod 2)$.

Received by the editors October 13, 1981 and, in revised form. March 11, 1982.

1980 Mathematics Subject Classification. Primary 57Q99: Secondary 57R95.

'The authors are members of the G.N.S.A.G.A. 
An Euler pair is a pair of polyhedra $(P, Q)$ such that (1) $\forall x \in P \backslash Q, \chi(\operatorname{lk}(x, P))$ $\equiv 0(\bmod 2) ;(2) \forall x \in Q, \chi(\operatorname{lk}(x, Q)) \equiv 0(\bmod 2) ;(3) \forall x \in Q, \chi(\operatorname{lk}(x, P)) \equiv 1$ $(\bmod 2)$.

RemarKs. (1) An Euler space is a cycle (without boundary).

(2) An Euler pair $(P, Q)$ is $n o t$, in general, a cycle with boundary (if $\operatorname{dim} P=n, Q$ may not be of dimension $n-1)$.

(3) Note that the definition of an $n$-cycle is slightly different from the usual one which requires also each simplex of $K$ to be the face of an $n$-simplex of $K$. However, a cycle as we defined it naturally carries a fundamental class (which is a cycle in the usual sense) as follows:

Let $P=|K|$ be an $n$-cycle. The fundamental class $\tilde{P}$ of $P$ is the polyhedron obtained by taking all the $n$-simplexes of $K$ (together with their faces). Note that, if $P$ is connected, then $\tilde{P} \hookrightarrow P$ is a representative of the generator of $H_{n}\left(P ; \mathbf{Z}_{2}\right) \cong \mathbf{Z}_{2}$.

In order to show the kind of arguments used, we first prove an "abstract" version of the stated result, that is

THEOREM 1. Let $P$ be an $n$-cycle. Then there exists an Euler polyhedron $P^{\prime}$ such that $P^{\prime} \supset P$ and $\operatorname{dim}\left(P^{\prime} \backslash P\right)<n$.

Proof. Let $P=|K|$ and assume that $K=T^{(1)}$, that is, $K$ is the first barycentric subdivision of another triangulation $T$ of $P$. Set

$$
Q=\overline{\{A \in K: \chi(\operatorname{lk}(A, K)) \equiv 1(\bmod 2)\}} .
$$

$Q=|H|$ is a subpolyhedron of $P$ and $\operatorname{dim} Q<n-1$ (as $P$ is a cycle).

(a) Assume $\operatorname{dim} Q=0$. Then $Q$ consists of a finite number of points $v_{1}, \ldots, v_{h}$ and $(P, Q)$ is an Euler pair. Let $Z$ be the 1 -skeleton of $K$; then (for the properties of the barycentric subdivision) $Z$ is a 1 -cycle with boundary the 0 -skeleton of $H$, that is, $Q$ itself (see [A], Propositions 1 and 2, and the subsequent remark). Thus $h$ is even and we can form $P^{\prime}=P \cup_{Q} \Gamma$, where $\Gamma$ is any 1-cycle with boundary $Q$.

(b) The general case. Let $d=\operatorname{dim} Q(0<d \leqslant n-2)$. We prove first of all that $Q=|H|$ is a $d$-cycle. Let $A$ be a $(d-1)$-simplex of $H$ and $B_{1}, \ldots, B_{h}$ the set of $d$-simplexes of $H$ such that $B_{i}>A$. If $C$ is a simplex of $R=\operatorname{lk}(A, K)$, then $C * A \in K$ and $\operatorname{lk}(C, R)=\operatorname{lk}(C * A, K)$ (here * denotes the join operation). Since $\operatorname{dim}(C * A)=\operatorname{dim} C+d, \chi(\operatorname{lk}(C, R))$ is always even, except for the vertices $v_{1}, \ldots, v_{h}$ such that $v_{i} * A=B_{i}$. Then, by the case (a), $h$ is even, which means that $Q$ is a cycle.

Now we can form $P_{1}=P \cup_{Q} \Gamma$, where $\Gamma$ is any $(d+1)$-cycle with boundary $Q$, for example the cone on $Q . P_{1}$ is not necessarily an Euler space; however, if $B$ is a $d$-simplex of $H, \operatorname{lk}\left(B, P_{1}\right)=1 \mathrm{k}(B, P) \amalg\{$ odd number of points $\}$, so that $Q_{1}$ $=\overline{\left\{A \in P_{1}: \chi\left(\operatorname{lk}\left(A, P_{1}\right)\right) \equiv 1\right\}}$ is a subpolyhedron of dimension $\leqslant(d-1)$ in $P_{1}$; by iterating the argument we obtain the required Euler space $P^{\prime}$.

Note that the hypothesis that $P$ is a cycle is necessary; see, for example, the following Figure 1.

The difficulty which arises in the general case is essentially to prove that $Q$ is now a boundary in the ambient manifold. 


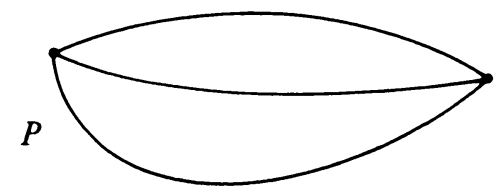

FIGURE 1

THEOREM 2. Let $M$ be a closed m-manifold and $P$ a cycle of dimension $n<m$ in $M$. Then there exists a subpolyhedron $P^{\prime}, P \subset P^{\prime} \subset M$, such that $P^{\prime}$ is an Euler space and $\operatorname{dim}\left(P^{\prime} \backslash P\right)<n$.

Proof. Let $Q$ be defined as in the previous theorem and $(L, K, H)$ be a triangulation of $(M, P, Q)$ which we assume, for the sake of simplicity, to be the first barycentric subdivision of another triangulation of $(M, P, Q)$ (see remark below).

Claim. $Q$ is a boundary in $P$.

(Note that this has already been proved in the case $\operatorname{dim} Q=0$.) Let $d=\operatorname{dim} Q$; let $N$ be the simplicial neighbourhood of $H^{(1)}$ in $K^{(1)}, \dot{N}$ the boundary of $N, p$ : $N \rightarrow Q$ the simplicial retraction and $\dot{p}=p \mid \dot{N} .(\overline{P \backslash N}, \dot{N})$ is an Euler pair; therefore (again by [A, Proposition 1]), if $Z$ denotes the $(d+1)$-skeleton of $\overline{P \backslash N}$ and $S$ denotes the $d$-skeleton of $\dot{N}$ (both with respect to $K^{(1)}$ ), we have that $Z$ is a $(d+1)$-cycle with boundary $S$. Let $f=\dot{p} \mid S ; f$ is a simplicial map and we want to show that its degree is odd. Let $\sigma \in H^{(1)}$ be a $d$-simplex and $A \in H$ such that $\sigma \subset A$; we must prove that $\#\left\{\right.$ simplexes in $\left.f^{-1}(\stackrel{\circ}{\sigma})\right\}=\#\left\{d\right.$-simplexes in $\left.\dot{p}^{-1}(\stackrel{\circ}{\sigma})\right\}$ is odd; as

$$
\begin{aligned}
\#\{B \in K: A<B\} & =\#\{\text { simplexes } C \text { of } \operatorname{lk}(A, K)\} \\
& \equiv \chi(\operatorname{lk}(A, K)) \equiv 1 \quad(\bmod 2),
\end{aligned}
$$

it is enough to show that, for each $B>A, \#\left\{d\right.$-simplexes in $\left.\dot{p}^{-1}(\stackrel{\circ}{\circ}) \cap \dot{B}\right\}$ is odd. Let $B>A$; then $B=A * C$ and $\dot{p} \mid \dot{N} \cap B: \dot{N} \cap B \rightarrow A$ is obtained by the pseudoradial projection from $C$.

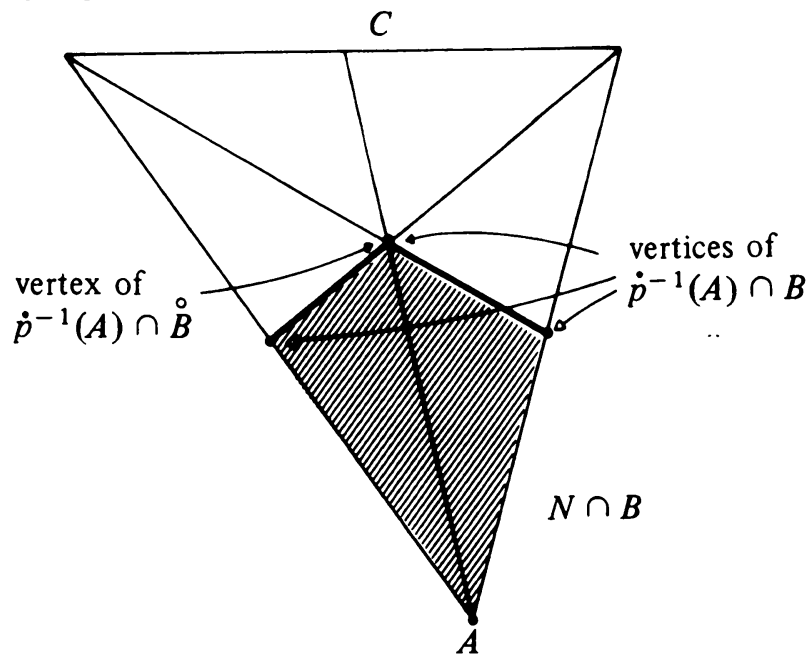

Figure 2 


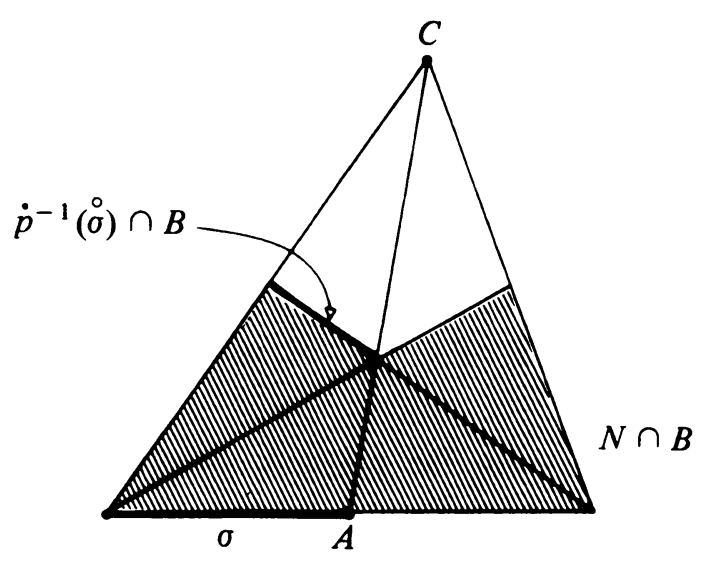

FIGURE 3

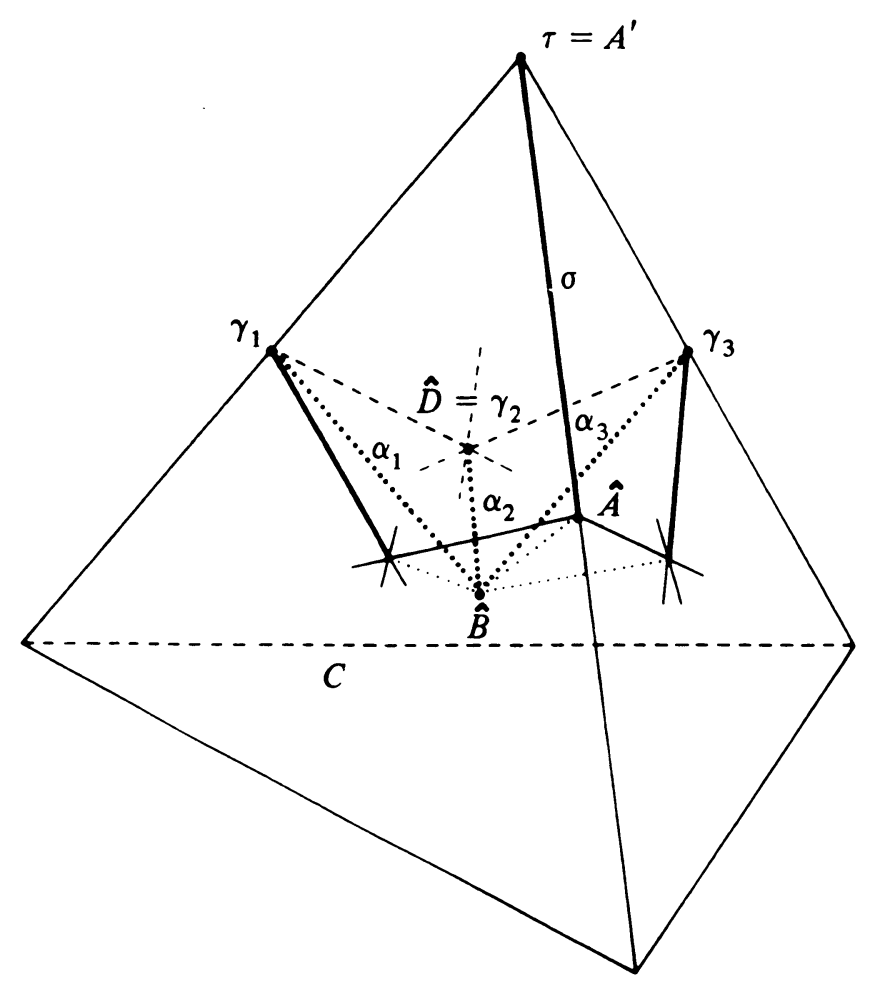

FiguRE 4

Note that, if $\operatorname{dim} A=0, \#\left\{\right.$ vertices of $\left.\dot{p}^{-1}(A) \cap \dot{B}\right\}=1$ and $\#$ vertices of $\left.\dot{p}^{-1}(A) \cap B\right\}=\#\left\{\right.$ vertices of $C$ in $\left.K^{(1)}\right\} \equiv 1(\bmod 2)$ (see Figure 2$)$; while, if $\operatorname{dim} C=0$ (so that $B$ is a cone over $A$ with vertex $C$ ), \#\{d-simplexes in $\left.\dot{p}^{-1}(\stackrel{\circ}{\sigma}) \cap \stackrel{\circ}{B}\right\}$ $=\#\left\{d\right.$-simplexes in $\dot{p}^{-1}($ o $\left.) \cap B\right\}=1$ (see Figure 3). In general, if $\sigma=\hat{A} * \tau$, let $A^{\prime}$ 
be the face of $A$ containing $\tau$ and $D=C * A^{\prime}$. Then, if $\alpha$ is a $d$-simplex in $\dot{p}^{-1}(\stackrel{\circ}{\sigma}) \cap \dot{B}$, necessarily $\alpha=\hat{B} * \gamma$, where $\gamma$ is a $(d-1)$-simplex in $\dot{p}^{-1}(\tau) \cap D$ (see Figure 4). In order to conclude by induction, we have to show that also $\#\{d$-simplexes in $\left.\dot{p}^{-1}(\stackrel{\circ}{\sigma}) \cap B\right\}$ is odd. But, if $C^{\prime}$ varies over the faces of $C$, and $B^{\prime}=A * C^{\prime}$, then

$$
\begin{aligned}
\#\left\{d \text {-simplexes in } \dot{p}^{-1}(\stackrel{\circ}{\sigma}) \cap B\right\}= & \#\left\{d \text {-simplexes in } \dot{p}^{-1}(\stackrel{\circ}{\sigma}) \cap \dot{B}\right\} \\
& +\sum_{C^{\prime}<C} \#\left\{d \text {-simplexes in } p^{-1}(\stackrel{\circ}{\sigma}) \cap \dot{B}^{\prime}\right\},
\end{aligned}
$$

By induction, all the terms of this sum are odd; moreover, their number equals $\#\left\{C^{\prime}: C^{\prime} \leqslant C\right\} \equiv 1(\bmod 2)$. Thus $f: S \rightarrow Q$ is an odd degree map, so that the mapping cylinder $C_{f}$ is a $(d+1)$-cycle in $P$ with boundary $S \amalg Q$ and $Q^{\prime}=Z \cup_{S} C_{f}$ is the required cycle with boundary $Q$. This proves the claim.

In order to prove the theorem, it is enough now to put $Q^{\prime}$ transverse to $P$ in $M$ relatively to $Q$ (see [RS, Theorem 5.3]). In this way we get a cycle $Q^{\prime \prime}$ in $M$ with boundary $Q$ and such that $\operatorname{dim}\left(Q^{\prime \prime} \cap P\right) \leqslant d+1+n-m \leqslant d$. Form $P_{1}=$ $P \cup_{Q} Q^{\prime \prime} ; P_{1}$ is an $n$-cycle in $M$ and, if $A$ is a $d$-simplex in $P_{1}$, then

$$
\operatorname{lk}\left(A, P_{1}\right)= \begin{cases}\operatorname{lk}(A, P) \amalg\{\text { odd number of points }\} & \text { if } A \in Q, \\ \operatorname{lk}(A, P) & \text { if } A \in P \backslash Q^{\prime \prime}, \\ \operatorname{lk}\left(A, Q^{\prime \prime}\right) & \text { if } A \in Q^{\prime \prime} \backslash P, \\ \operatorname{lk}(A, P) \amalg\{\text { even number of points }\} & \text { if } A \in Q^{\prime \prime} \cap P .\end{cases}
$$

In each case $\chi\left(\operatorname{lk}\left(A, P_{1}\right)\right) \equiv 0$, so that $Q_{1}=\overline{\left\{A \in P_{1}: \chi\left(1 \mathrm{k}\left(A, P_{1}\right)\right) \equiv 1\right\}}$ has dimension $\leqslant(d-1)$ and we can iterate the argument as before until we get an Euler space $P^{\prime}$.

REMARK. As regards the choice of the triangulation, what we need is only that the simplicial neighbourhood $N$ of $Q$ in $P$ (with respect to $K^{(1)}$ ) is in fact a regular neighbourhood; therefore, any triangulation $(K, H)$ such that $Q$ is full in $P$ would be enough (see [RS] for a definition of full).

COROLlARY. Every homology class $z \in H_{n}\left(M, \mathbf{Z}_{2}\right)$ can be represented as the fundamental class of an Euler subpolyhedron of dimension $n$ in $M$.

ADDENDUM. With respect to the problem stated in the introduction (that is, to represent $\mathbf{Z}_{2}$-homology classes of a real algebraic manifold by algebraic subvarieties), since this paper was written we have proved the following (see [BD]):

For each $d \geqslant 11$, there exists a compact smooth manifold $V$ and a class $z \in$ $H_{d-2}\left(V, \mathbf{Z}_{2}\right)$ such that, for any homeomorphism $h: V \rightarrow V^{\prime}$ between $V$ and a real algebraic manifold $V^{\prime}, h_{*}(z) \in H_{d-2}\left(V^{\prime}, \mathbf{Z}_{2}\right)$ cannot be represented by an algebraic subvariety of $V^{\prime}$. 


\section{REFERENCES}

[A] E. Akin, Stiefel-Whitney homology classes and bordism, Trans. Amer. Math. Soc. 205 (1975), 341-359.

[BT] R. Benedetti and A. Tognoli, Remarks and counterexamples in the theory of real algebraic vector bundles and cycles, Lecture Notes, Springer, Berlin and New York (to appear).

[RS] C. P. Rourke and B. J. Sanderson, Introduction to piecewise-linear topologv, Ergebnisse der Math. und ihrer Grenzgebiete, Band 69. Springer-Verlag. Berlin and New York, 1972.

[S] D. Sullivan, Combinatorial invariants of analytic spaces, Proc. Liverpool Singularities Symposium. I, Lecture Notes in Math., vol. 192, Springer-Verlag, Berlin and New York, 1971.

[BD] R. Benedetti and M. Dedò, Counterexamples to representing homology classes by real algebraic subvarieties up to homeomorphism (to appear).

Istituto Matematico, via F. Buonarroti 2, 56100 PiSa, Italy 